\title{
Geographical and Temporal Characteristics of Inter-POP Flows: View from a Single POP
}

\author{
Supratik Bhattacharyya, Christophe Diot, Nina TAFt \\ Sprint ATL, Burlingame, CA. 94010 USA \\ JORJETA JETCHEVA \\ Carnegie Mellon University, Pittsburg, PA. USA
}

December 21, 2001

\begin{abstract}
.
In this paper, we study traffic demands in an IP bacbkone, identify the routes used by these demands, and evaluate traffic granularity levels that are attractive for improving the poor load balancing that our study reveals. The data used in this study was collected at a major POP in a commercial Tier-1 IP backbone. In the first part of this paper we ask two questions. What is the traffic demand between a pair of POPs in the backbone? How stable is this demand? We develop a methodology that combines packet-level traces from access links in the POP and BGP routing information to build components of POP-to-POP traffic matrices. Our analysis shows that the geographic spread of traffic across egress POPs is far from uniform. In addition, we find that the time of day behaviors for different POPs and different access links also exhibit a high degree of heterogeneity. In the second part of this work, we examine commercial routing practices to assess how these demands are routed through the backbone. We find that traffic between a pair of POPs is engineered to be restricted to a few paths and that this contributes to widely varying link utilization levels. The natural question that follows from these findings is whether or not there is a better way to spread the traffic across backbone paths. We identify traffic aggregates based on destination address prefixes and find that this set of criteria isolates a few aggregates that account for an overwhelmingly large portion of inter-POP traffic. We demonstrate that these aggregates exhibit stability throughout the day on per-hour time scales, and thus form a natural basis for splitting traffic over multiple paths to improve load balancing.
\end{abstract}

\section{INTRODUCTION}

Internet backbones continue to grow at explosive rates, fueled by the bandwidth demands of new applications and by the advent of faster access technologies. To accomodate such growth while preserving the robustness of the network, most IP backbone operators have chosen a simple approach to traffic engineering: overprovisioning. Overprovisioning is the adopted approach because very little information exists today about the dynamics of the traffic in an IP backbone. This is primarily due to the lack of measurement infrastructure and techniques for collecting and processing data from backbones. To address this deficiency, we study traffic traces collected at a Point of Presence (POP) in a commercial Tier 1 IP backbone network. A passive monitoring system is used to collect packet-level traces on a number of access links within the POP [1]. The data is then analyzed offline in order to understand the dynamics of traffic entering the backbone at this POP. We describe a methodology for extracting information about routing and traffic flow within the backbone. This method- ology forms the basis for building components of POP-toPOP level traffic matrices, which are key to studying a variety of traffic engineering and routing issues. We investigate how much can be said about the traffix matrix just from the data collected at a single POP.

It has been observed $([2,3,4])$ that obtaining information about traffic patterns in both time and space is critical for most traffic engineering functions. Traffic engineering typically operates on long time-scales such as minutes, hours, weeks or longer. Examples of traffic engineering functions include dimensioning, provisioning, route optimization, where to best add new customer links, load balancing policies, designing POP architectures, and selecting failover strategies. The particular application determines the level of information needed about traffic patterns. Since IP networks do not typically generate feedback state information, traffic engineering has to rely on traffic measurements [2]. It has been observed that simulation data cannot provide substitutes [5]. Therefore collecting traffic measurements spanning multiple hours in order to build 
network-wide views of the traffic flows is central to being able to efficiently engineer an IP backbone.

A network-wide view is typically expressed in the form of a traffic matrix ([6, 3, 7]). A variety of information can be represented in it. For example, the traffic volume captured in the matrix can refer to any level of flow granularity. A traffic matrix also has an associated time granularity that specifies the measurement interval over which bandwidth measurements were averaged. The choice of exactly what is represented in the matrix depends upon the traffic engineering task to be performed with this matrix. In a POP-toPOP traffic matrix the rows represent ingress POPs and the columns represent egress POPs. Since our data was collected at a single POP in our network, we build one row of a POP-to-POP traffic matrix. Due to the cost of such equipment, the enormous difficulties involved in deploying the equipment in commercial backbones and the scarce availability of this backbone data, even this component of a traffix matrix constitutes a significant amount of useful information.

We decompose and study this data along a number of different dimensions. The work in this paper can be viewed as a search of answers to the following questions, each of which logically follows from the next. In the first part we ask, what is the traffic demand between a pair of POPs? How stable is this demand? The traffic matrix compiled in this part only describes the demand or how much traffic wants to go from one POP to another; it says nothing about how the traffic is routed. Thus in the second part we ask, how are these demands routed in our commercial backbone? Are link utilization levels similar throughout the backbone? Our observations from these two parts are that traffic is highly non-uniform in a geographic sense yet the ranking of POPs (in terms of volume) remains fairly stable in time; and that few routes are used and link utilization levels vary widely throughout the backbone. These findings motivate the third part which asks, is there a better way to spread the traffic across the paths? And at what level of granularity should this be done?

For the first part, we proceed to study the partition of traffic throughout the backbone as follows. We examine incoming traffic at a single POP at different levels of granularity. First, we analyze the spatial characteristics of POPlevel traffic. We discover a large disparity in the spatial distribution of the ingress POP's traffic across the egress POPs. Second, we break up the ingress POP's traffic according to access link, and examine the spatial distribution of the traffic from specific types of access links across the egress POPs. We find that the same disparity appears at this level of granularity. We compare the access links and find that they behave differently from one another with respect to three metrics considered. For example, we find that one cannot isolate a single probability distribution to characterize the geographical fanout of the traffic from access links. We also examine time of day behavior of the traffic at both the POP-level and access link level. We find that egress POPs can be ranked roughly into three categories (large, medium and small) based on the amount of traffic they receive from the ingress POP, and that POPs generally remain in the same category through the entire day. A stronger statement can be made about many of the POPs - if they are ranked by the volume of traffic they receive, they maintain their ranking throughout the day. We also find that at night the overall traffic load is reduced by only 15-50\% depending upon the access link.

For the second part of our work, we begin by checking whether or not overprovisioning has led to a disparate use of resources on a network-wide basis. By examining both SNMP data, we do indeed find that the amounts of excess link capacities are inequitably distributed throughout our backbone. We then study IS-IS routing behavior to understand how IS-IS is engineered to influence path selection, and how the routing impacts the link utilization levels. We find that the backbone is carefully engineered using IS-IS weights to restrict traffic between POP pairs to a few paths even though many alternate paths exist.

In the third part of our study, we return to our traffic data to assess at what granularity level it is desirable to do load balancing. We want to determine a traffic granularity that defines a unit of flow (or stream) that could be rerouted on an alternate path. Having examined our data at both the POP-level and the access-link-level, we now study the data at the granularity level of destination address prefixes. We find that a small number of these aggregate streams, called elephants, generate a large fraction of the total traffic, while a large number of these streams, called mice, generate a small fraction of the total traffic. The elephants and mice phenomenon has been observed before in Internet traffic at the inter-AS level [4], at the level of multipoint demands from one router node to a set of router nodes [3] and in the Internet as it was many years ago [8]. Here we demonstrate this phenomenon at the granularity level of specific prefixes. We also demonstrate the stability of these aggregates throughout the day. The stability of these elephants makes them well-suited as a basis for routing traffic on alternate paths and thus improving the load balance in the backbone.

The rest of the paper is organized as follows. Our measurement infrastructure is briefly presented in Section 2. Section 3 describes a methodology for building a POP-toPOP view of traffic flow across the backbone, based on observations at an ingress POP. Our technique makes extensive use of BGP and IS-IS routing information. The space and time characteristics of traffic at the POP-level and the access link level are analysed in Section 4. In Section 5 we study IS-IS routing in order to understand how routing practices influence the partition of traffic across the backbone. In Section 6 we aggregate the traffic based on destination address prefixes, and demonstrate the existence of the elephants and mice phenomenon at this granularity level. We analyze properties of these aggregates and discuss their application to load balancing. Section 7 dis- 


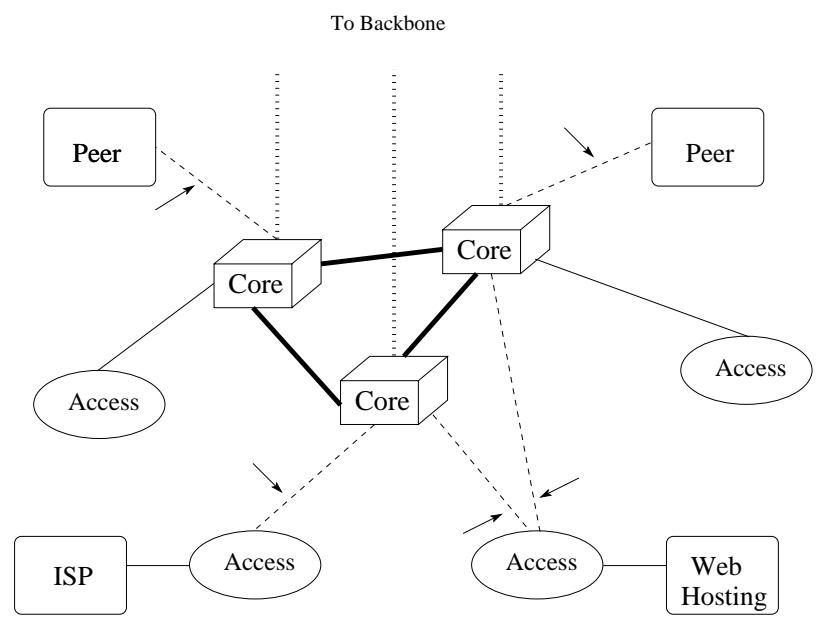

Figure 1: Monitored POP Links/Architecture of a POP

\begin{tabular}{|c|c|c|c|}
\hline Link & $\begin{array}{c}\text { Trace Length } \\
\text { (hours) }\end{array}$ & Trace Size & $\begin{array}{c}\text { \# Packets } \\
\left(10^{6}\right)\end{array}$ \\
\hline Peer 1 & 16 & $51 \mathrm{~GB}$ & 853 \\
\hline Peer 2 & 24 & $47 \mathrm{~GB}$ & 794 \\
\hline Web Host 1 & 19 & $51 \mathrm{~GB}$ & 853 \\
\hline Web Host 2 & 13 & $51 \mathrm{~GB}$ & 853 \\
\hline Tier 2 ISP & 8 & $17 \mathrm{~GB}$ & 284 \\
\hline
\end{tabular}

Figure 2: Summary of Data

cusses related work, and Section 8 discusses some of the implications of our results and identifies directions for future work. This paper is an extended version of the results published in [9].

\section{MEASUREMENT INFRASTRUCTURE}

The data used for this study was gathered from an operational IP backbone using the passive monitoring infrastructure described in [1]. The backbone topology consists of a set of nodes known as Points-of-Presence (POPs) connected together by high bandwidth backbone links. Each POP also locally connects customers through access links, ranging from large corporate networks to regional ISPs and webservers. Peering at a POP is provided either through dedicated links to another backbone (private peering) or through public Network Access Points (NAPs). Each POP has a two-level hierarchical structure (Figure 1). At the lower level, customer links are connected to access routers. These access routers are in turn connected to the backbone routers. The backbone routers provide connectivity to other POPs and to the peers. The backbone links connecting the POPs are optical fibers with bandwidths of $2.5 \mathrm{Gbps}$ (OC48). They carry IP traffic using the Packet-over-SONET (POS) protocol. The exterior and interior gateway protocols for the backbone are Border Gateway Protocol (BGP) and IS-IS respectively.

The infrastructure developed to monitor this network consists of passive monitoring systems that collect packet traces and routing information on the links located between the access routers and the backbone routers, or on the peering links. The monitoring systems tap onto the selected link using optical splitters, and collect the first 44 bytes of every packet on these links. Every packet record is timestamped using a GPS clock signal which provides accurate and fine-grained timing information. The format of the packet record is as follows.

- GPS timestamp : 8 bytes

- Size of record : 4 bytes

- Size of POS frame : 4 bytes

- HDLC header : 4 bytes

- IP packet header : 44 bytes

BGP tables were downloaded from one router ${ }^{1}$ in the POP once per hour during the time the packet traces were collected. In this study, we used data from five access links, collected on August 9, 2000, starting at 10:00am US Pacific Standard Time (UTC-7). The arrows in Figure 1 indicate the monitored links used in this study. The table in Figure 2 provides a summary of our traces. The traces are of different lengths because packets were collected until the hard disk in each monitoring system filled up. Therefore heavily loaded links filled up the disk faster than lightly loaded links. We have collected many other traces during August and September 2000. The results in this paper have been verified against one other day. We present the data from a single day to avoid overloading the paper with excessive graphs.

\section{Methodology}

In this section we explain how we constructe the row of our POP-to-POP traffic matrix representing our backbone traffic. This row in the traffic matrix corresponds to data that originates at the monitored POP (i.e., the ingress POP) and leaves the network through each of the other egress POPs (including itself). To do this, we need to map each packet received at the monitored POP, to the egress POP through which it leaves the network. All backbone routers participate in the BGP protocol and exchange information with each other about routes learned from routers external to the network, called external peers. This information is kept in the BGP table of each router and can be used to determine the last egress router for each packet destination. However, information on mapping an egress router to an egress POP is not readily available and has to be derived from the values of standard BGP attributes.

We start by illustrating how we map a packet to an egress POP through an example (Figure 3). Consider a packet with a destination address of 1.1.1.1. Suppose that

\footnotetext{
${ }^{1}$ all routers in the POP have the same view of BGP routes
} 


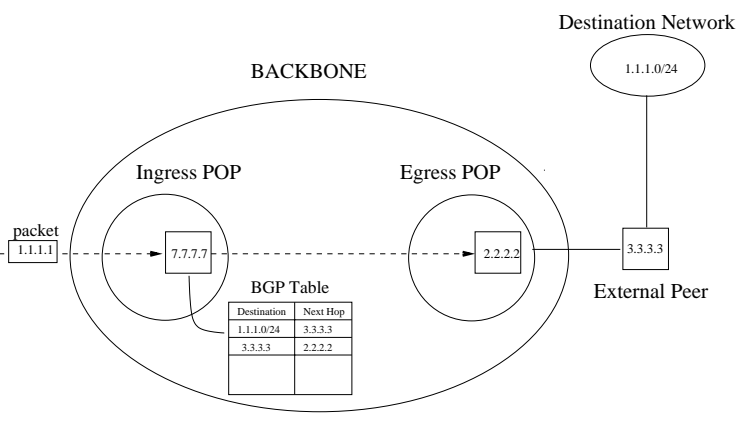

Figure 3: Example of destination to egress POP mapping

the BGP table at the ingress router (7.7.7.7 in the figure) for this packet, identifies the destination subnet for this packet as 1.1.1.0/24. The BGP table entry at the ingress router, which corresponds to this subnet contains a Next-Hop entry which is typically the address of the external BGP peer from which a border router in our backbone first learned the route to the destination and injected it into the I-BGP mesh. This border router is in the egress router for destination 1.1.1.1, since it is the router that packets for subnet 1.1.1.0/24 need to go through in order to reach the external peer on their way to the destination network. Suppose the address of the border router is 2.2.2.2 and that the address of the external peer recorded in the Next-Hop entry in the BGP table is 3.3.3.3 ${ }^{2}$. The BGP table at 7.7.7.7 also contains an entry for 3.3.3.3 (or the subnet containing it), whose Next-Hop field identifies the address of the border router, i.e. the egress router for this packet. To find the egress POP for the packet destination, we use the BGP Community Id attribute which is an identifier shared by all routers at a POP. This attribute is recorded in each BGP table entry and identifies the egress POP for all destinations that map to that entry. In our example, the Community Id allows us to identify the POP to which the egress router 2.2.2.2 belongs.

However, that are many cases when the Community Id attribute for a route is not set to an identifier that specifies the egress POP (due to internal policies) for the BGP entry it belongs to. In such cases, we extract the Originator attribute for the route announcement to a given NextHop. The Originator attribute value corresponds to the address of a router in our backbone. In the above example, the router 2.2.2.2 would be the Originator for the route to 3.3.3.3. Querying the BGP table returns the Community Id attribute associated with the Originator, and hence the POP at which the Originator is located. This POP is the egress POP associated with the Next-Hop that we are interested in (3.3.3.3 in our example).

Note that there are a few cases, when BGP attributes fail to reveal the POP name. In these cases, we perform

\footnotetext{
${ }^{2}$ Typically a BGP table will contain a number of alternate paths for a destination subnet. However we consider here only the route chosen as the "best" or "preferred" route based on BGP policies and attribute values.
}

a Traceroute to the Next-Hop router address associated with the BGP entry for the packet destination. We can extract the name of the last hop router within the backbone from the output of Traceroute, and derive the identity of the POP from the name. The name of each router at a POP contains a sub-string that is derived from the name of the city in which the POP containing the router is located (for example, a router's name in a POP in Miami would contain the string mia).

The complete algorithm for determining the egress POPs for destination networks in the BGP table is described in Figure 1. The BGP tables were downloaded from core routers using the show ip bgp facility. For ease of exposition we introduce the functions $\operatorname{Nexthop}(R)$, $\operatorname{community}(R)$ and originator $(R)$, which return the values of the Next-Hop address, Community Id attribute and the Originator attribute respectively for a route $R$.

Recall that the BGP tables we used were collected once an hour, a time-scale on which they have been observed to be relatively stable [10]. The number of unique Next-Hop entries in each table was on the order of a few thousands. Of these, about $98 \%$ were resolved to egress POPs using BGP attributes, and the rest were resolved using Traceroute. Overall, more than $99 \%$ of the destination networks in the BGP tables were resolved to egress POPs using our technique.

Once we obtain a mapping of destination networks to egress POPs, we can apply it to the packet traces to determine the traffic flowing from the monitored POP to each other POP. This task is analogous to the problem of performing lookups on packets arriving at a router to determine the egress port. For this purpose, we used the IP lookup technique described in [11]. This technique uses an LC trie-based longest prefix match and a software implementation is publicly available ${ }^{3}$. We modified this software to perform a longest prefix match on packets in a trace using our destination-to-POP map. The output consists of per-egress-POP packet traces. These can be further analyzed to understand the dynamics of traffic between any two POPs in the network. We have developed tools to subdivide the traffic between two POPs based on various criteria such protocol number, destination prefix, etc. Tools have also been designed and implemented, to study the variation of traffic on different timescales. These analysis tools were used to compute all of the results presented in this paper.

\section{Traffic in Space and Time}

\subsection{GeOgRAPHIC SPREAD}

POP-Level. We look at the geographical spread of traffic demands across egress POPs, or fanout, first at the POP-

\footnotetext{
${ }^{3}$ http://www.nada.kth.se/ snilsson/public/soft.html.
} 


$$
L=\{\}
$$

For every destination $d$ in the BGP table do

next_hop $=\operatorname{NextHop}(d)$.

if $\quad($ next_hop $\notin L)$

$$
L=L \cup\{\text { next_hop }\}
$$

endif

endfor

For every next_hop in $L$ do

$\operatorname{POP}($ next_hop $)=0$.

extract $R A_{\text {next_hop }}$, the route advertisement for next hop.

pop_id $=\operatorname{community}\left(R A_{\text {next } \_ \text {hop }}\right)$.

if pop_id $\in\{$ list of known POP identification numbers $\}$ then

$\operatorname{POP}\left(n e x t \_h o p\right)=p o p \_i d$.

else

$O=$ originator $\left(R A_{\text {next_hop }}\right)$.

pop_id $=\operatorname{community}(O)$.

if $p_{0} i d \in\{$ list of known POP identification numbers $\}$ then $\mathrm{POP}=$ pop $\_i d$.

endif

if $(\mathrm{POP}($ next_hop $)==0)$ then

run traceroute to next_hop.

egress_router $=$ last Sprint router on the traceroute path to next $\_$hop.

egress_router_name $=$ well known name for router $r$.

Parse egress_router_name to determine the id of the POP egress_router belongs to.

$\mathrm{POP}($ next_hop $)=$ pop_id . endif

endfor

For the entry for every destination $d$ in the BGP table do next_hop $=\operatorname{NextHop}(d)$.

$\operatorname{POP}(d)=\operatorname{POP}($ next_hop $)$.

endfor

Table 1: Destination to Egress POP Mapping Algorithm. 
level and then on an access-link level. Since most of our traces span from 13 to 24 hours each (depending upon the link), we are also able to study the time of day behavior for these demands. Our goal in this section is to classify the basic behaviors we observe into a few categories, and to understand the range of behaviors that can occur. We are also interested in comparing different types of access links and different egress POPs to see if and where commonalities lie.

First we consider the traffic demands on all five access links together as one input stream. Note that this constitutes a significant portion of the input traffic at our monitored POP. Given the variety of access links chosen, this is also highly representative of the total input traffic entering the POP. The monitored POP is henceforth refered to as the ingress $\mathrm{POP}$.

We use the methodology described in the previous section to classify all the packets in a trace by their egress POPs. We then determine the total number of bytes headed towards each egress POP using the packet length information in the IP header of each packet record. This gives us the fanout of traffic demands by volume (Figure 4). The values presented in this figure are bandwidth values that were averaged over the duration of the entire trace for every link. This fanout constitutes the row on our POP-toPOP traffic matrix.

For the purposes of display we have organized the POPs into 3 groups: the west, midwest and east regions of the United States. The monitored POP is located in the west coast of the US. For proprietary reasons the POPs are only identified with numbers. Within each of the 3 regions the ordering is arbitrary and does not have any geographic signifigance.

We observe that there are two POPs that are clearly dominant, and receive a large amount of traffic (over 35 Mbps). Among the remaining POPs about half receive quite a small amount of traffic (under $5 \mathrm{Mbps}$ ) and the other half receive a moderate amount of traffic (10-20 Mbps). Our data suggests that ingres POPs could be roughly categorized as large, medium and small, where (i) roughly the same number of POPs fall into the small and medium categories and only a few fall into the large category; and (ii) each category carries approximately twice the volume of the category below it. This simple characterization will prove useful in our interpretation of other data below. (We will discuss the stability of these characteristics in the next subsection.)

Often in simulation environments, researchers assume a traffic matrix. In the past, in absence of data, the most common model is, given a source, pick a destination at random according to a uniform distribution. This histogram reveals that such an approach does not at all match Internet behavior. Moreover, thinking about how the Internet is designed, it is easy to understand why we see this nonuniform behavior. First, one would expect that some POPs would sink higher traffic demands than others because of their geographic location. For example, dominant POPs are expected to be located on the two coasts of United States because this is typically where international trunks terminate, and because the coasts are more heavily populated than the center of the country. Secondly, one would expect this distribution to exhibit a significant degree of variation. The volume of traffic an egress POP receives (from other ingress POPs) depends upon a large number of factors, such as the number and type, of customers and servers attached to it. Similarly, the amount of traffic an ingress POP generates can also vary enormously depending upon the number and type, of customers and servers, on its access links. Thus we expect the inter-POP flows to vary dramatically from one to another, and to depend on the (ingress POP, egress POP) pair. Our final observation on this fanout is that the ratio of the largest POP-pair to the smallest POP-pair is approximately 40:1.

Recall that the data in our fanout plot describes the amount of traffic exiting Sprint's network at a given POP; however this only includes traffic that originated at the monitored POP. In order to see whether our observations about traffic egressing POPs hold independently of the particular ingress POP monitored, we did the following. We collected the SNMP data (i.e., average link utilization levels) from all links at all POPs. We can thus compute the average amount of flow entering and exiting any given POP based on the links attached to the POP. (Recall that the SNMP data does not given any information about the ingress or egress point of the traffic.) There are 3 kinds of links exiting a POP: customer links, peering links and inter-POP links. To assess the amount of traffic egressing a POP we only consider the first two types of links. Thus Figure 6 describes the rate of traffic egressing a given POP, summing the average rate of all customer and peering links exiting the POP. For each link the rate is averaged over a $21 / 2$ month period, as indicated in the figure. Data was unavailable for POPs numbered 10 and 16.

We see from this figure, that the relative sizes of POPs remains unchanged. In other words, the large POPs (namely numbers 4, 7, 8, 12 and 14) remain large over the many months considered. Also, the small POPs (namely numbers 5, 69) remain small over time. The others could be categorized as medium sized POPs. There are, of course, a few odd data points. For example, POP \#11 experienced a large growth in the January-March 2001 period. Otherwise it remains consistenly in the medium category. Note that the ordering of POP sizes in this figure need not correspond to the ordering in Figure 4, because the data in Figure 4 represents the data originating from a single POP while the data in Figure 6 includes the data originating from all POPs. We also note that the difference between the largest POP and the smallest can be quite dramatic. Finally we point out that a few POPs experienced a reduction in size during the last $21 / 2$ month period considered. We suspect that this is due either to the movement of customer links or to the addition of new links elsewhere in the net- 


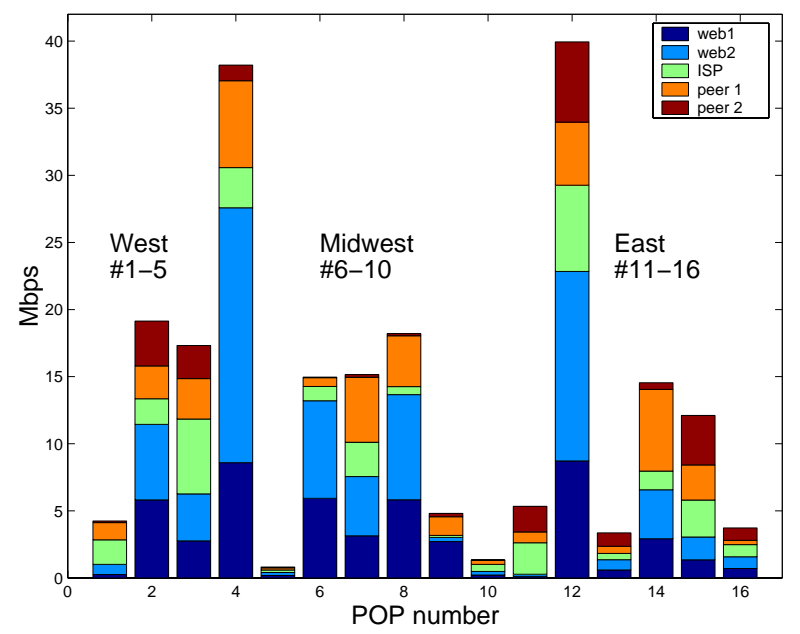

Figure 4: Fanout of 5 Access Links Combined

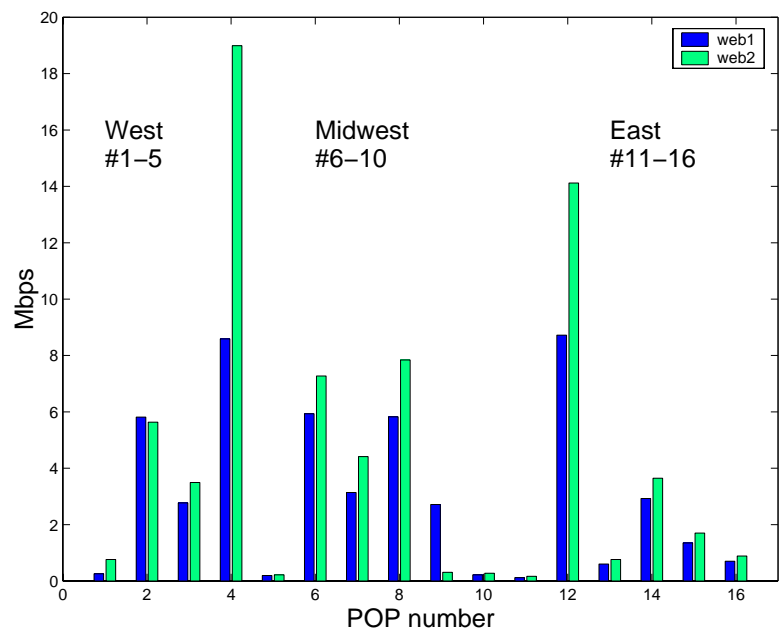

Figure 5: Fanout of Web Host Links

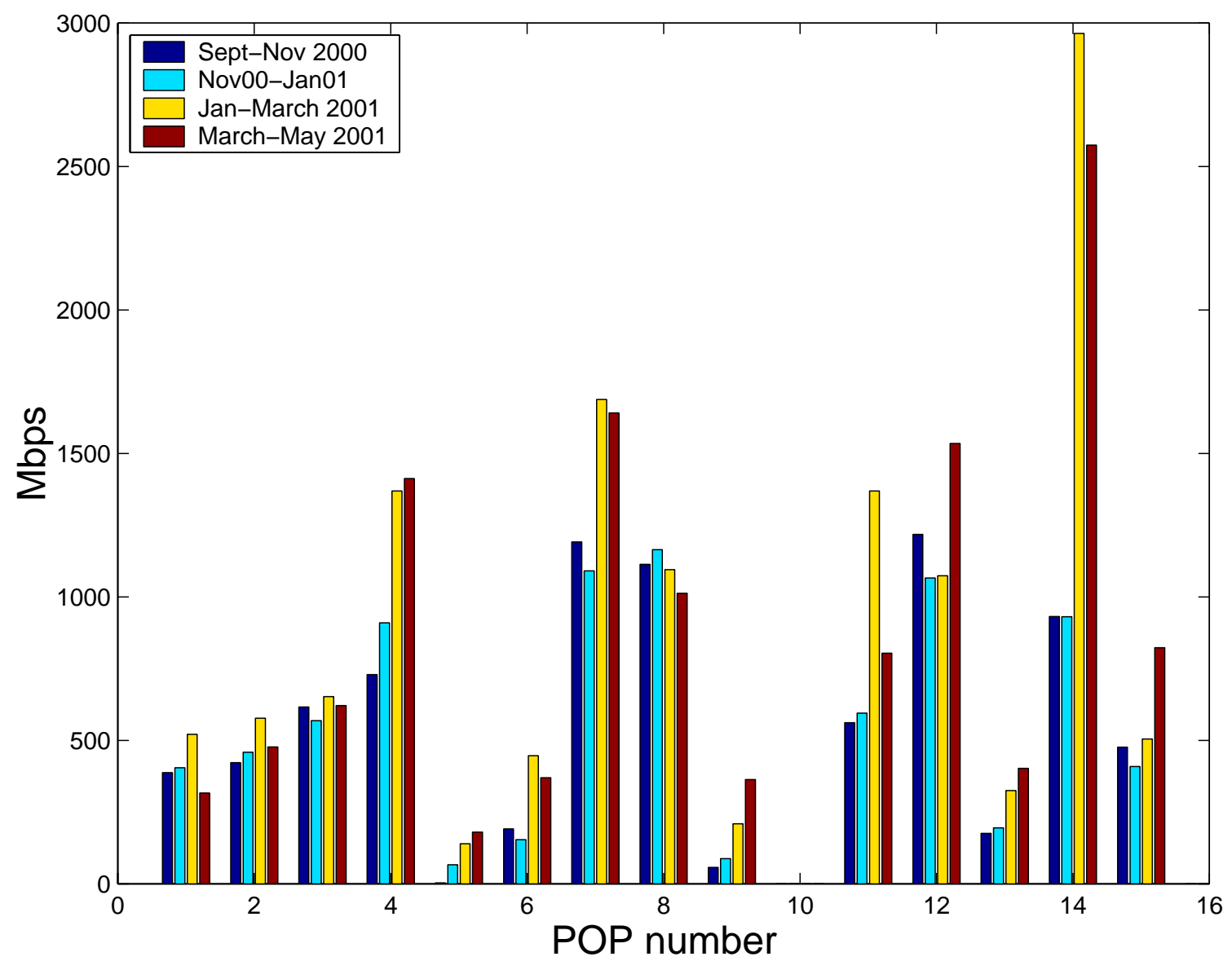

Figure 6: Average Amount of Data Egressing Sprint's Network. SNMP Data for all POPs. 


\begin{tabular}{|c|c|c|c|c|c|}
\hline $\begin{array}{l}\text { Ingress } \\
\text { Link }\end{array}$ & $\begin{array}{c}\text { peer } \\
\# 1\end{array}$ & $\begin{array}{c}\text { peer } \\
\# 2\end{array}$ & ISP & $\begin{array}{c}\text { webhost } \\
\# 1\end{array}$ & $\begin{array}{c}\text { webhost } \\
\# 2\end{array}$ \\
\hline $\begin{array}{l}\text { volume } \\
\text { (Mbps) }\end{array}$ & 40 & 22 & 32 & 50 & 70 \\
\hline $\max / \min$ & 13 & 50 & 13 & 35 & 63 \\
\hline
\end{tabular}

Table 2: Comparison of Access Links

work.

Access-Link-Level. For the purposes of bandwidth prediction, the (ingress POP, egress POP) pair might represent a level of granularity that is too coarse for accurate traffic estimation. It is natural to hypothesize that the access links at the ingress POP may differ from one another, and may affect the traffic flowing to each egress POP differently. We thus next consider the fanout of traffic at the ingress POP on a per-access-link-type basis. To compare these links we considered three metrics: (i) the total volume of traffic per link (summing across all egress POPs); (ii) the $\mathrm{max} / \mathrm{min}$ ratio of the average bandwidth headed towards an egress $\mathrm{POP}^{4}$ and (iii) the distribution among the egress POPs. The total volume and max/min ratios are given in Table 2. We see that the access links differ from one another with respect to these simple measures that span a range of values.

Figure 5 demonstrates that the rough categorization we applied to egress POPs at the POP-level (i.e., the large, medium and small categories) continues to hold at the level of input access link type. (The same is true for the peering links, however we exclude the fanout plot due to space restrictions.) In other words, a very small number (between 1-3) of POPs receive a large amount of traffic and the rest of the POPs are evenly split between the medium and small categories. To compare the fan-out of the different access links numerically, we normalize and convert the fan-out information per link into a probability distribution. Let $b_{i j}$ denote the average bandwidth that access link $i$ sends to POP $j$ during one day. For a given link $i$, the probability that a byte of data chosen at random gets sent to POP $j$ is given by $P_{i}(j)=b_{i j} / \sum_{j} b_{i j}$. The density curves for each of the five links is given in Figure 7. The ordering of the POPs here is different than in the previous graphs, and thus it no longer represents an east/midwest/west organization. This ordering was selected to try to isolate a pattern.

To facilitate the discussion, we use the term popularity of a POP to refer to the likelihood that a byte of data

\footnotetext{
${ }^{4}$ In computing the max/min ratio we ignored the three smallest POPs for a given access link because there were typically a few POPs that receive a negligible amount of traffic and this creates ratios that are not representative.
}

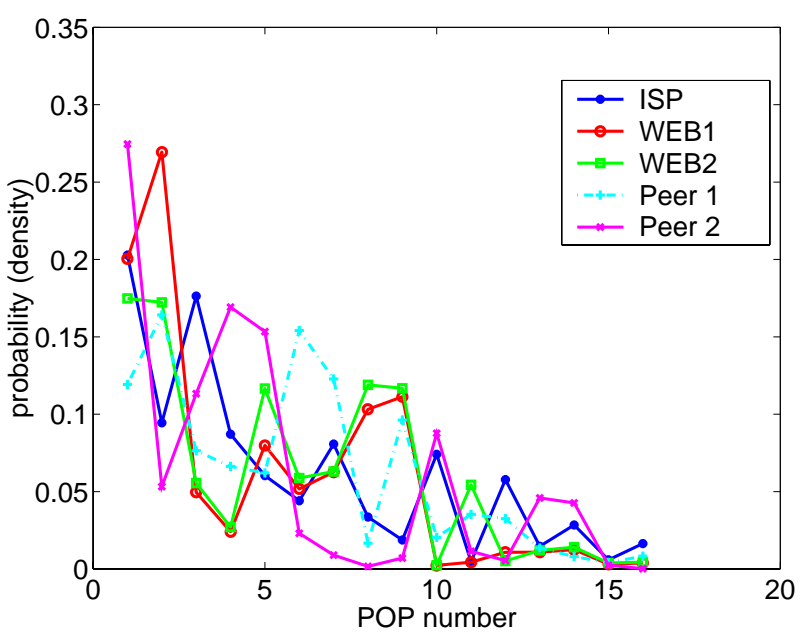

Figure 7: Probability density of POP fanout per access link type

from an ingress access link will be sent to that egress POP. On the one hand, we see a few similarities among the five links. The first two POPs are the most popular among all the access links. POPs \#11-16 are fairly unpopular for all links. For all other POPs, the popularity ordering jumps around quite a bit for each link. For example, the likelihood that a packet on a given link will choose POP \#4 can vary from 0.02 to 0.17 . This graph indicates that POP \#3 is most likely to be chosen by our ISP link, POPs \#4 and \#5 are most likely to be chosen by the peer 2 link, POPs $\# 6$ and \#7 are most likely to be chosen by the peer 1 link, and \#8 and \#9 by the second web host link. In general, for POP's \#3-\#10, the likelihood of being chosen can vary about $10 \%$. We believe that these differences are substantial and that the fanouts from the different links are sufficiently different so that one cannot conclude that there is a single underlying distribution that represents all the access links. Note that the categorization of egress POPs according to large/medium/small is the same for different access links. The access links differ in their geographic spread primarily in how they distribute traffic among the medium sized POPs.

We thus infer that when studying traffic demands for load balancing, and more generally, when designing bandwidth predictors for traffic engineering, the pair (ingress POP access link type, egress POP) should be explicitly accounted for rather than simply using the (ingress POP, egress POP) pair.

From this section, we conclude that in terms of geographic distribution there is a large disparity among the traffic sent to the egress POPs from a single ingress POP, and that the access links differ from one another significantly according to three different metrics. The exception is for the two web host access links; however, these two links carry traffic from the same client, which reinforces our notion that links generate different traffic demands based on their types. 


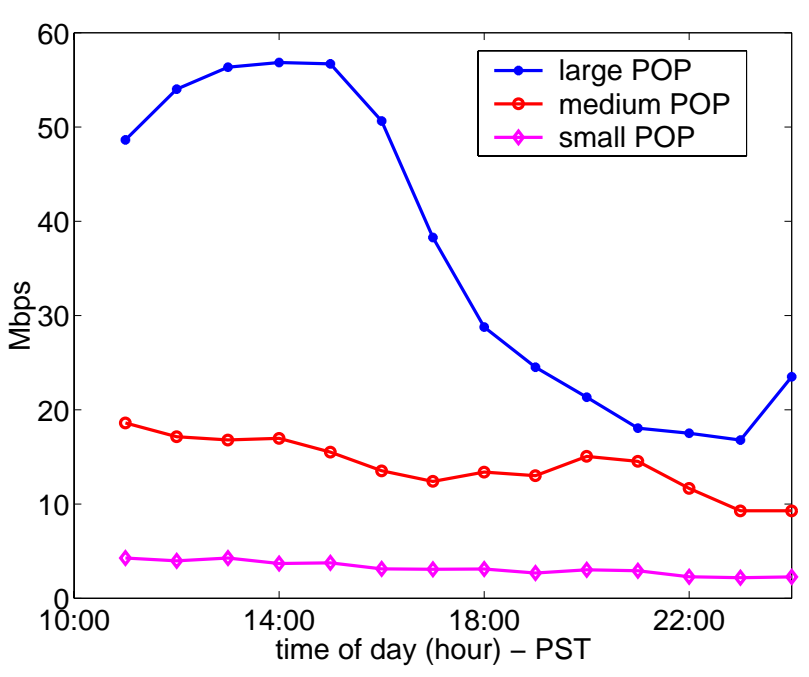

Figure 8: POP-level Time of Day

\begin{tabular}{|l|c|c|c|c|}
\hline trace & $\begin{array}{c}\text { peer } \\
\# 1\end{array}$ & $\begin{array}{c}\text { peer } \\
\# 2\end{array}$ & $\begin{array}{c}\text { web host } \\
\# 1\end{array}$ & $\begin{array}{c}\text { web host } \\
\# 2\end{array}$ \\
\hline$\%$ reduction & $30 \%$ & $30 \%$ & $50 \%$ & $16 \%$ \\
\hline
\end{tabular}

Table 3: Night vs. Day Traffic

\subsection{TIME OF DAY BEHAVIOR}

In the previous section, the fanouts we examined were computed based on day-long averages. In order to examine the consistency of the fanout throughout the day, we look at inter-POP flows on an hourly basis. In Figure 8 we consider just four of our input links (because the 5th has too few hours) and examine the behavior throughout the day of three representative POPs, one in the large category, one in the medium and one in the small. First, we observe that the large POP is the most volatile, that the medium POP experiences a long slow small decline, and that the small POP remains fairly stable. We examined a number of other POPs and found this behavior to be consistent of POPs within their respective categories. Second, we observe that during the day the distinction between large, medium and small remains, whereas at night the large and medium POPs become less distinguishable.

Figure 8 indicates that some POPs do not experience much decrease in traffic volume at night, while others (particularly those in the large category) do. When we considered traffic volume between 10 AM to 6 PM (daytime) and 6 PM to 6 AM (nightime) separately, we found that the nighttime peak traffic is about $30 \mathrm{Mbps}$, about half of the daytime peak. The average percent reduction on a per-link basis is shown in Table 3. The table indicates that the average volume of night time traffic is anywhere from $15-50 \%$ less than the average volume of day time traffic. This is surprising since it is counter to the widely held belief that traffic in the backbone reduces by a few factors at night.
This may arise from an increase in international traffic and night-time backup activities.

We now examine time of day behavior (Figures 9-12) at the access link level to examine the variations and if our previous observations hold at the access link level as well. In order to compare different access links, we provide a separate figure for each of four access links (identified in the figure caption). For each access link, we plot the hourly bandwidth averages for six different POPs, hence each curve corresponds to a single egress POP. Some of the curves on these graphs exhibit a sharp drop around 2:00am. This is due to maintenance activities at the POP. From these four plots we observe the following:

- A number of POPs have traffic that remains fairly constant throughout the day.

- A number of POPs experience a long slow decline of loading throughout the day.

- The most popular POPs are usually the most volatile.

- If we were to rank the POPs by volume received, then most of the POPs (excepting the few large ones) maintain their rank throughout the day.

- POPs can experience an increase at night (see Figure 11 and 12).

These observations are interesting in that they reveal counter-intuitive things about busy periods. Our experience from telephone networks leads us to expect peak period behaviors in time-of-day plots. These figures reveal that some POPs do not experience any busy periods, some POPs experience one busy period, and others can experience two.

We see that the category that an egress POP falls into can depend upon the access link. For example, consider POP \#13. On the two peering links, this POP is a small one. On the two web hosting links, it would be considered a medium one. This indicates that the fraction of traffic than an egress POP draws from an ingress POP depends upon the number and type of input access links. An alternative way to see this is given in Figures 13 and 14. In these plots we compare the traffic destined for a single egress POP originating from each of the access links. This illustrates that an egress POP's behavior can differ dramatically depending upon which access link on an ingress POP it receives traffic from. For some access links, an egress POP receives a roughly constant amount of traffic while for others its traffic experiences peaks and dips over the course of the day. Thus the incoming traffic on an egress POP is directly dependent upon the type of access link at the ingress POP. 


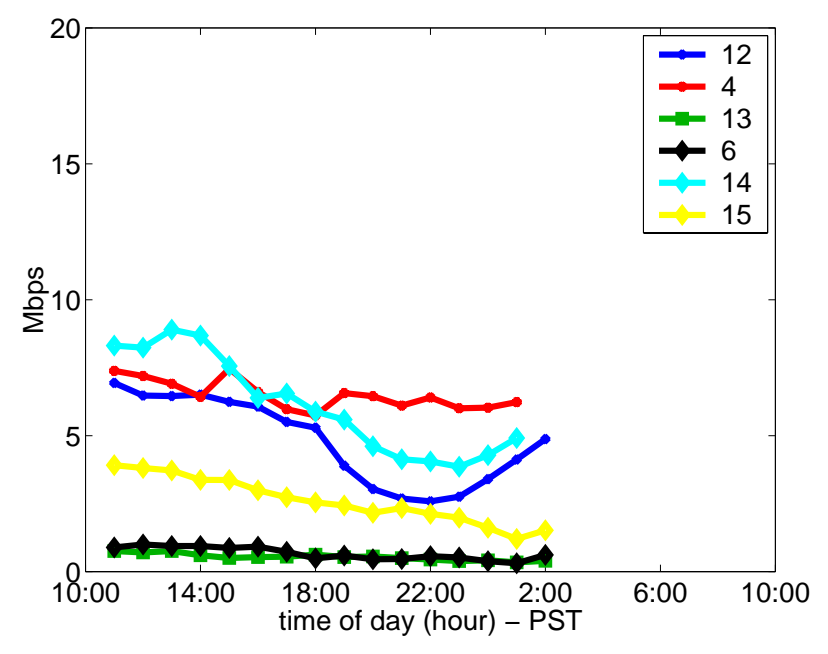

Figure 9: Peer 1

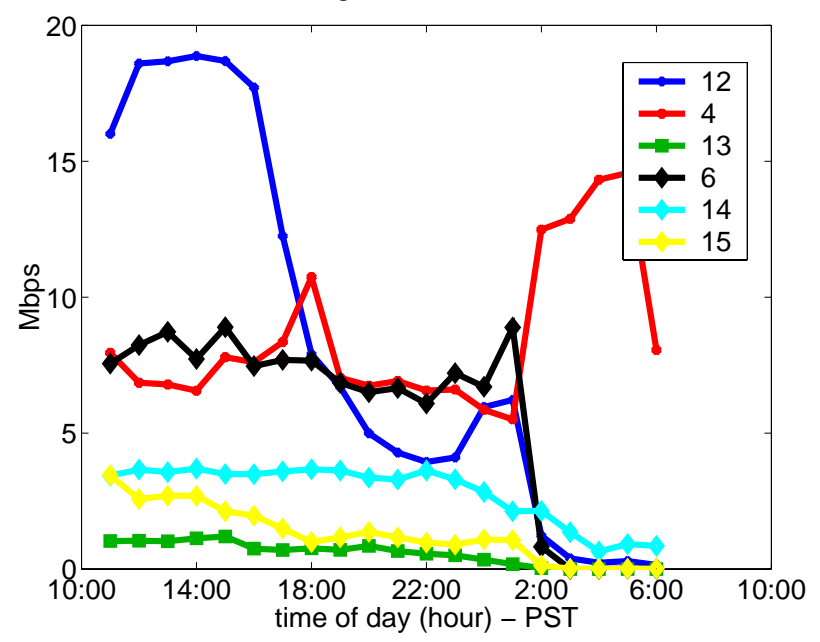

Figure 11: Web Host Link \#1

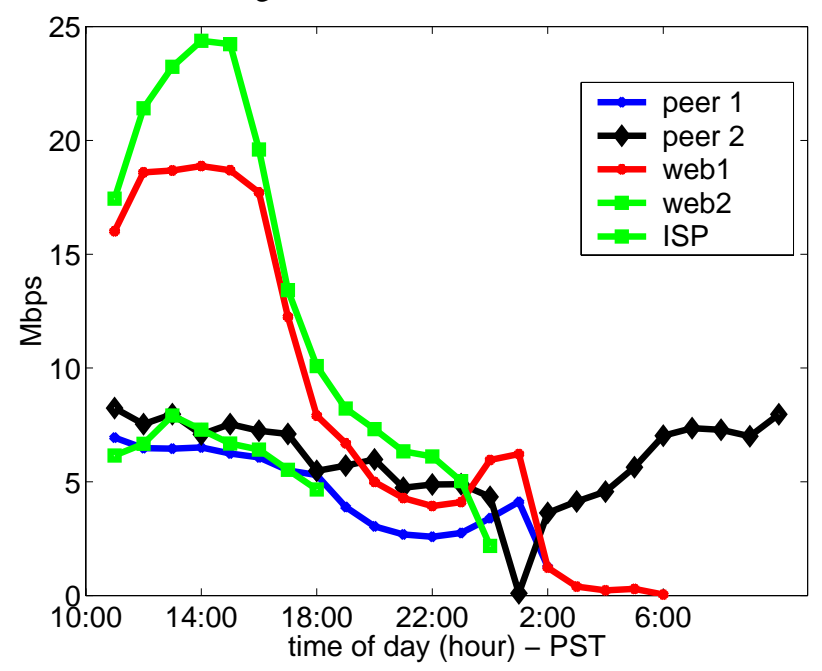

Figure 13: East Coast POP



Figure 10: Peer 2

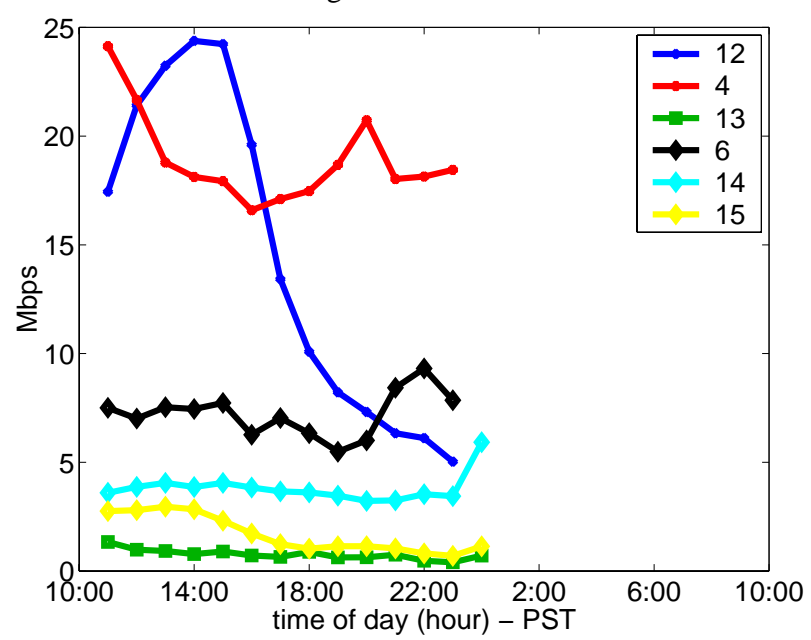

Figure 12: Web Host Link \#2



Figure 14: West Coast POP 


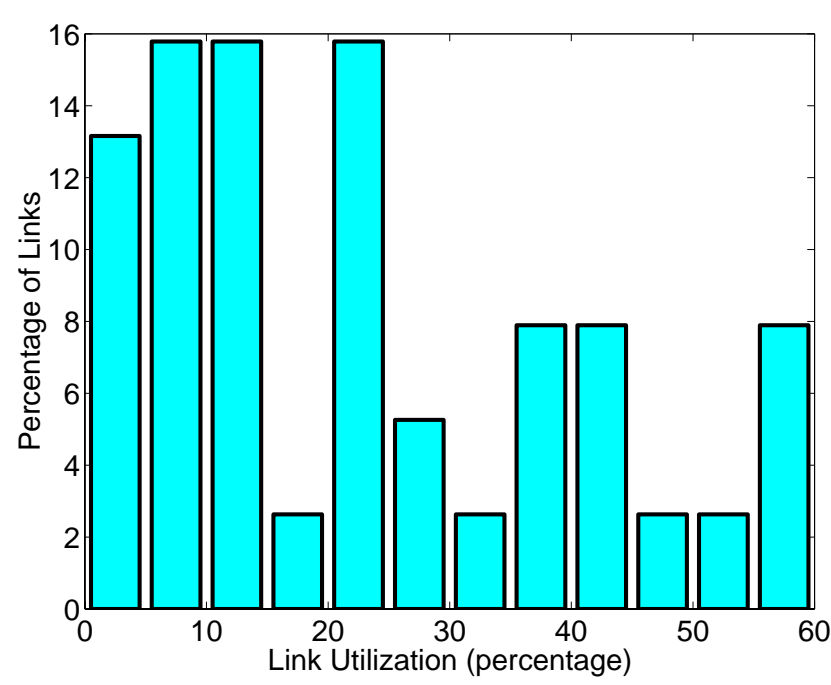

Figure 15: Average Link Load Levels

\section{ObSERVATIONS About IS-IS Routing IN THE BACKBONE}

In the previous section we examined properties of traffic demands, i.e. how much traffic wants to go from one end of our network to another end. This says nothing about how that demand is routed through our network. The interior gateway protocol used for routing traffic in a backbone has a direct effect on link load levels. We thus study how IS-IS is configured in our backbone to understand how traffic demands are routed through the backbone, and thus how IS-IS may contribute to any imbalance in link load levels. Before doing so, we first present the average link load levels from all the backbone links in our network (collected via SNMP data). Figure 15 provides a histogram of this data, averaged over an entire day. We find that the majority of the links have an average utilization under $25 \%$, and that the link utilization levels can vary from $3 \%$ to $60 \%$. This histogram reveals (i) the extent of link underutilization and (ii) the extent of the disparity in the utilization levels of the backbone links.

IS-IS is a link state routing protocol where each link in the network is assigned a weight. Every router broadcasts information about its connectivity to other links and routers, including the costs of paths to reach them. The cost of any path is the sum of the weights of all the links that constitute that path. Once a router has received path cost information from all other routers, it constructs a map of the entire network and then routes data to every other router using the minimum cost path.

We start our study of IS-IS routing by constructing a detailed topology map of the backbone based on precise information about all the links in the backbone and the IS-IS weights assigned to them. This enables us to determine the minimum cost path (as would be seen by IS-IS) between these router pairs. We found the following. A packet's path has three components: the path through the ingress POP, the path through the backbone and the path through the egress POP. Typically, a packet enters an ingress POP at an access link and traverses a number of hops within the ingress POP. Recall that POPs themselves usually contain a large number of routers. Once a packet leaves the ingress POP, it traverses a number of intermediate POPs, but usually travels through a single core router at each of these. At the egress POP, the packet again traverses multiple hops before it exits the backbone.

We find that the backbone is carefully engineered using IS-IS weights. IS-IS weights on links between POPs are chosen such that traffic between an ingress-egress POP pair is constrained to a few paths across the backbone. Moreover these paths are usually partially overlapping and have nearly the the same costs. The choice of which path to take among the few options is thus heavily determined by the number of hops the packet has to traverse at the ingress and egress POPs and the link weights within these POPs. In other words, we find that the POP links weights and POP architecture have a strong influence on how equal cost paths through the backbone are used.

Let us illustrate these observations with a case study of traffic flow between a particular ingress-egress POP pair in the backbone (Figure 16). The ingress and egress POPs ( $I$ and $E$ respectively) are expanded to show the generic POP architecture ${ }^{5}$ while all intermediate POPs are represented as single nodes. The names of all core routers start with the letter "R", access routers start with an "A", and intermediate POPs start with an "H". A packet traverses only core routers at the intermediate POPs. For reasons of confidentiality, all link weights have been adjusted by a constant value.

Consider a packet that enters the network at router $A 1$ and leaves the backbone at router $A 2$. There are two equal cost paths through the backbone, namely $(H 1, H 2, R 4)$ and $(H 1, H 3, R 4)$ indicated via thick lines in the figure. Using the backbone segment $(H 1, H 2, R 4)$, a packet can travel over either of the following full paths: $(R 1, R 3, H 1, H 2, R 4)$ or $(R 2, R 3, H 1, H 2, R 4)$. These two equal cost paths differ only by the access link traversed after leaving $\mathrm{A} 1^{6}$. This type of an architecture, with two access links connecting an access router to two different core routers within a POP is very common, and is meant for fault tolerance and load balancing. We found many instances of a pair of access links, interconnecting a single access router to multiple core routers, having the same weights.

Because of these equal cost links, IS-IS will split incoming traffic evenly over these two links. Unfortunately, this kind of load balancing is only effective within the POP,

\footnotetext{
${ }^{5}$ In reality the POP architecture is much more complex.

${ }^{6}$ The existence of the other equal cost backbone segment does not change the point we are trying to illustrate.
} 


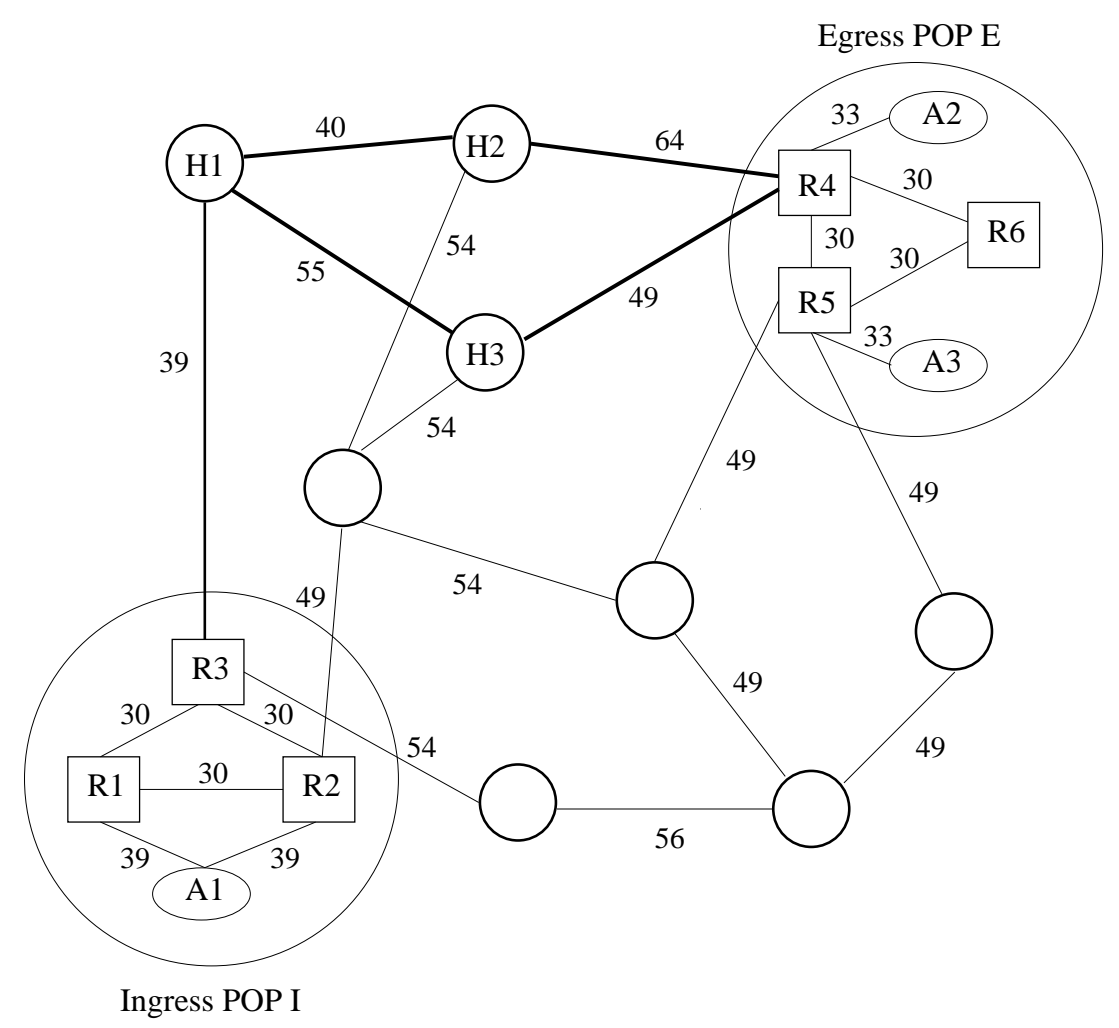

Figure 16: ISIS-Routing in the Backbone : A case study

since in either case packets from $A 1$ will exit the POP at $R 3$. In our study of POP-level traffic below, we will indeed see traffic streams for which this happens. This kind of load balancing is extremely sensitive to changes in the POP architecture or changes in IS-IS weights - if even one additional router is added along the path through $R 1$, all of the traffic from $A 1$ will be diverted to the path through $R 2$.

Let us next look at paths across the backbone between POPs. We find that a large number of alternate paths are available, but the assignment of IS-IS weights constrains packets from $A 1$ to take one of the two paths $(R 3, H 1, H 2, R 4)$ or $(R 3, H 1, H 3, R 4)$. IS-IS will split the traffic across these two equal cost paths at POP $H 1$. However, note that IS-IS load balancing works only if two paths have exactly the same cost - any difference in cost between two paths can result in all of the traffic being diverted to the lower cost path. We now describe a case where this limitation is evident.

The more common (and perhaps more interesting) case that we encounter is one where traffic is divided (though not equally) over two or more backbone paths based on the architecture of the egress POP. For ease of exposition, we do not present a separate case to illustrate this point; instead, we consider a slight modification to the case already under consideration. Suppose that the link from $H 3$ were connected to router $R 5$ instead of router $R 4$. When we calculate the path costs we see that all of the traffic to
$A 2$ (hence to all subnets reachable via $A 2$ will be routed via $H 2$ while all of the traffic to $A 3$ (hence to all subnets reachable via $A 3$ ) will be routed via $H 3$. In this case, there are no equal cost paths to either destination over which IS-IS can load balance. Furthermore, any division of traffic that happens on the inter-POP paths is static - a change in this division can be only effected through physical alterations to the configuration of POP $E$.

We have studied a number of cases of routing across the backbone and have reached a few conclusions about current IS-IS routing practices. First, the backbone is carefully engineered using IS-IS weights. This has two ramifications: (i) the weights are chosen such that traffic between any ingress-egress POP pair is restricted to only a few paths through the backbone; and (ii) the intra-POP link weights can heavily influence the path chosen to traverse the backbone. This has certain advantages such as ease of management. However, this approach can certainly drive up link utilization levels on these paths when the inter-POP traffic demands are heavy.

Second, we find that there do exist many alternate paths between POP-pairs, and that many of these paths are underutilized. This observation, plus our observations about the imbalance of link load levels, plus our findings about highly disparate inter-POP demands (Section 4) indicate that there is a lot of room for improved load balancing in today's networks. 
Improving the load balancing by manipulating IS-IS weights is not really a viable option for now. IS-IS does not have the capability to balance traffic across all of these paths unless they all have exactly the same cost. Currently, the IS-IS weights are handcrafted by network operations experts. It would be very difficult to assign link weights so as to create multiple equal cost paths between every POP pair in the network [12]. Moreover altering IS-IS weights has repercussions on the entire traffic matrix. We therefore search for an approach to load balancing that sets up policies that function on top of IS-IS.

Clearly using some of the underutilized paths, will often result in paths that have a larger number of hops than the minimum cost path. It is important to ensure that significant delays are not introduced to traffic that is rerouted on longer paths. We believe that this will not happen for two reasons. First, the backbone is highly meshed, and thus most alternate paths between an ingress-egress POP pair are likely to be only one or two hops longer than the min-hop path. Second, [1] shows that the average delay across routers in the backbone is on the order of a few milliseconds. Therefore, the additional delay that a packet will incur by traversing a few more routers is likely to be within acceptable limits.

\section{Traffic Aggregates for LoAd BAL-}

\section{ANCING}

In order to realize effective load balancing in the backbone, it is necessary to understand how traffic should be split over multiple alternate paths. In this section, we address this issue by examining techniques for creating aggregate traffic streams between (ingress link, egress POP) pairs. The aggregation of packets into streams can be based on a variety of criteria and can lead to streams with different levels of granularity. At the coarsest level, we can aggregate all the packets into a single stream. On the other hand, using the classic five-tuple of (source address, destination address, source port, destination port, protocol) leads to very fine-grained streams. The criteria used for creating traffic aggregates depends largely on the purpose of such aggregation. For example, when the goal is to provide different levels of service to different types of traffic, packets may be aggregated based on the (TOS) field or the protocol field in the packet IP header. Since we are interested in the routing of these aggregate streams across the backbone, it is natural to consider the destination address of packets as the basis for aggregation. Moreover routes are determined according the destination subnets (as advertised through BGP), each of which is an aggregate over a range of IP addrreses. Subnets in turn can be grouped on the basis of IP address prefixes. Therefore we consider destination address prefixes of different lengths as the basis for aggregating POP-to-POP traffic. For example, streams may be created based on an 8-bit destination address prefix, in which case all packets sharing the same first octet value for their IP address belong to one stream. We shall henceforth refer to such a stream as a $p 8$ stream. In general, when an $N$-bit prefix is used for aggregation, we refer to the aggregate stream as a $p N$ stream.

Aggregate traffic streams thus created would be assigned to different paths in order to balance the network load. Before adopting this approach to load balancing, we need to examine properties of these aggregates such as their traffic volume and their stability over the time interval for which such load balancing would be carried out.

We first consider $p 8$ streams and rank them in decreasing order of traffic volume (so that stream \#1 is the largest). Figure 17 shows the cumulative percentage of traffic of $p 8$ streams from the private peer access link and the webhost access link 1, respectively. For this access link, the traffic demand to three of the busiest egress POPs is presented. We see that for every egress POP pair, a few of the top-ranked flows account for an overwhelmingly large share of traffic. We have observed that this phenomenon is widespread across most other (ingress POP access link, egress POP) pairs. This brings us to an important result - the existence of a few very high-volume traffic streams, and many low-volume traffic streams in the backbone. We refer to the former as elephants and to the latter as mice. As mentioned in Section 1, the phenomenon of "elephants and mice" has been reported at other granularity levels in other traffic studies [4, 3, 8]. Here we demonstrate the existence of elephants and mice at specific IP destination address prefix levels in a commercial IP backbone.

The existence of elephants has important implications for traffic engineering in general, namely that in order to realize most of the benefits, we can focus primarily on engineering the network for the elephants. Many of the difficulties in providing quality of service in the Internet today stem from scalability issues. One cannot exert fine grained control because of scalability problems that arise with keeping too much state information. The elephants and mice phenomem means that one can try to exert more careful control on the elephants and that coarse control is sufficient for the mice. Although this has been observed before, we are not aware of any concrete suggestions or examples of using this traffic behavior to influence control. Elephants streams provide a basis for load balancing since once the elephants are identified, they can be rerouted over underutilized portions of the network. The criterion for identifying the elephants - destination address prefix - is simple enough for use in practice without new and complex protocols.

For simplicity of implementation, it is attractive to have a load balancing policy that is applicable over long time scales, such as a few hours, or even potentially throughout the day-time. Of course, our approach of load balancing via rerouting elephants, cannot be applied unless the ranking of elephants and mice remains fairly stable on these 


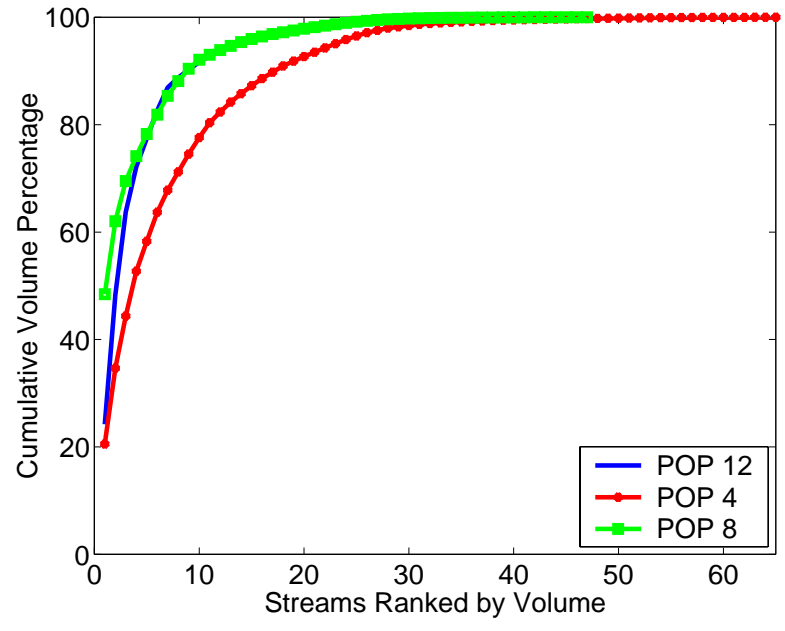

Figure 17: Distribution of traffic across p8 streams for Webhost access link 1

timescales. Figure 18 show the time-of-day variation of bandwidth for some of the elephants and mice to a busy POP from webhost 1 access link. In the graph, the onehour average of the bandwidths of these streams is plotted against time for 18 hours. We find that throughout this period, the elephants retain a large share of the bandwidth, and that they maintain their relative ordering. In other words, the elephants remain elephants and the mice remain mice. We have verified this behaviour for a large number of ingress-egress POP pairs. This result encourages us to focus our attention on just a few streams in the backbone for the purposes of load balancing.

Interestingly, we discover that the phenomenon of elephants and mice is recursive. In other words, if we consider a $p 8$ elephant stream, and then further subdivide it into substreams based on say a 16 bit prefix, then we find elephants and mice again among these substreams. In Figure 19 we consider the three largest elephants to each of the POPs 4 and 12 for the peer 1 access link, subdivide each into $p 16$ streams, rank them, and plot the cumulative volume for the ordered streams. Thus each curve in Figure 19 corresponds to the $p 16$ substreams from a single $p 8$ stream for a given POP. We find that 10 of the largest flows account for $80 \%$ or more of the bandwidth in every case. As with the $p 8$ streams, these $p 16$ elephants and mice exhibit stable behavior over many hours (figures omitted due to space considerations), even though the bandwidth of some of the elephants decreases substantially at night.

We further examine this recursive behaviour by taking some of the $p 16$ streams from the previous step and dividing them into substreams based on a 24-bit prefix. We find that although the elephants and mice phenomenon still exists, it becomes less pronounced as traffic becomes somewhat more uniformly distributed across streams (Figure 20). Although we investigate 1, 2 and 3 byte masks,

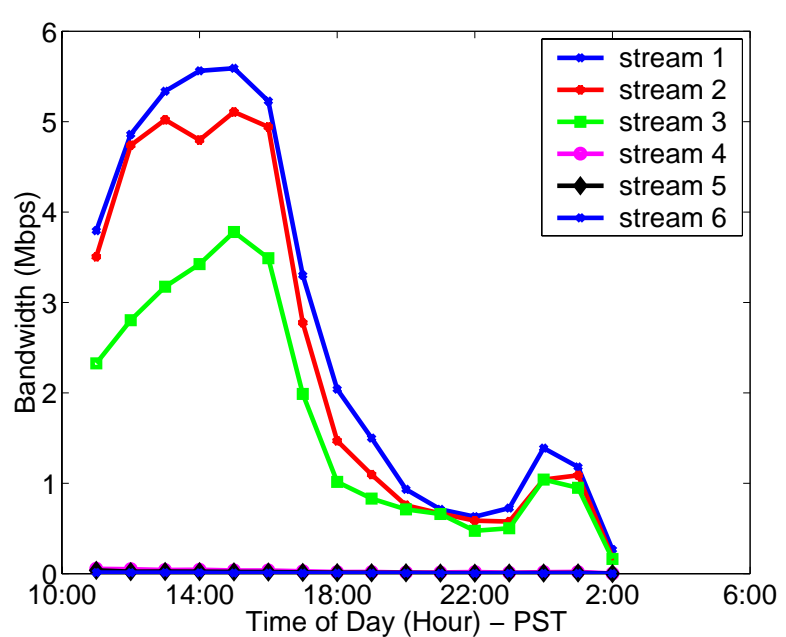

Figure 18: Time of day variations for $\mathrm{p} 8$ elephants and mice for Webhost access link 1

there is no particular association with Class A, B and C addresses that have become less meaningful with the advent of CIDR. In fact, we expect that this phenomenon will manifest itself at other prefix levels as well, certainly those between $8-24$, but probably less so at prefixes longer than 24.

A different way of studying the stability of elephants and mice is to look at the frequency and size of rank changes at a given prefix level. Suppose that we divide time into equal-sized slots and compute the average bandwidth for all the streams in each time slot. We can then rank the streams according to bandwidths and examine the change in rank of streams from one time slot to another. More precisely, let $R_{i}(n)$ be the rank of flow $i$ in time slot $n$, where $n=1,2, \cdots, N$ and $i=1,2, \cdots, M$. Let us define $\delta(i, n, k)=\left|R_{i}(n)-R_{i}(n+k)\right|$, where $1 \leq k \leq(N-n)$. For a given value of $k$, we examine the probability distribution for $\delta(\cdot, \cdot, k)$.

Figure 21 applies this technique for $p 8$ traffic streams between the peer 1 access link and POP 12 for an averaging interval of 30 minutes, and $k=1,2,4,6,8$ and 10 . The results show that most of the rank changes are small - in fact, rank changes of size 5 or less account for about $70 \%$ of the changes. Moreover, this is true for rank changes over different time intervals ranging from 30 minutes to five hours. Besides the obvious implication for load balancing, this result has a powerful implication for building traffic matrices. It provides a direction for predicting the rank of a stream at a future time based on the current rank. Development of such prediction techniques requires extensive analysis and sophisticated statistical models, and is beyond the scope of the current paper.

This distribution contains all the elephants and mice mixed together. To isolate the rank change behavior of the elephants alone, we checked the ranking of particular ele- 




Figure 19: Distribution of traffic across p16 streams for peer $1 \mathrm{ac}-$ cess link

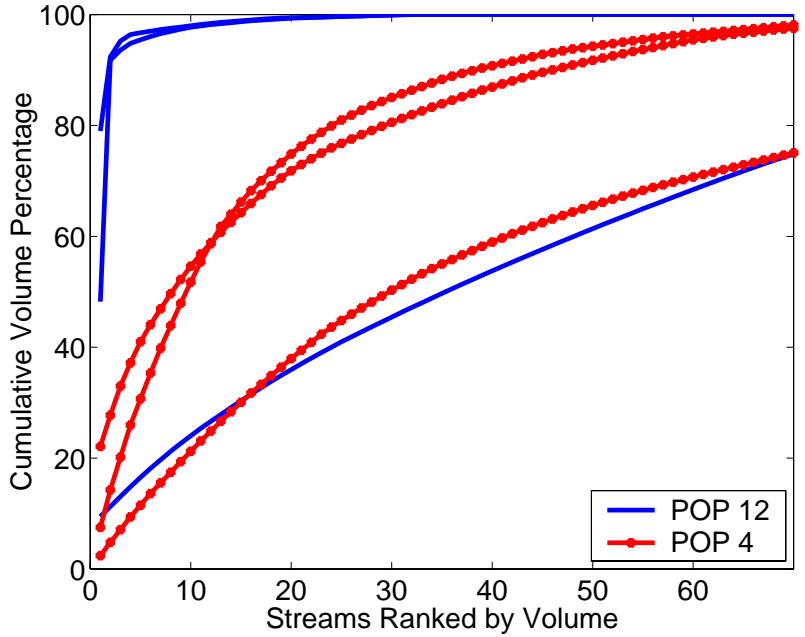

Figure 20: Distribution of traffic across p24 streams for peer 1 access link

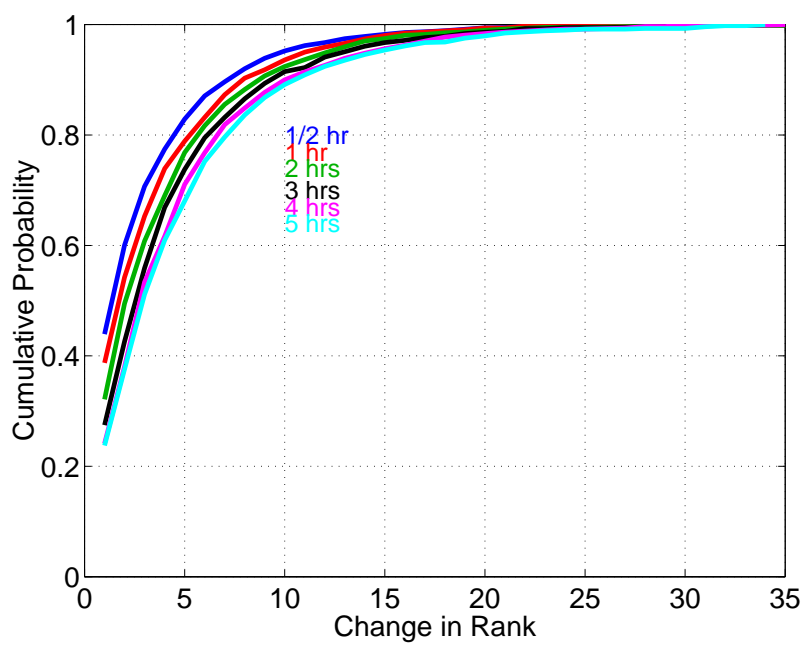

Figure 21: Cumulative distribution of rank changes for $\mathrm{p} 8$ traffic streams between peer 1 access link and a busy egress POP 
phants over entire traces. We found that $70 \%$ of the top 15 elephants remain among the top 15 streams throughout the day. We verified this behavior for a number of $\mathrm{p} 8$ streams and a number of egress POPs.

In summary, we demonstrate the existence of the elephants and mice phenomenon at the granularity of $p 8, p 16$ and $p 24$ prefixes. We show that this phenomenon is recursive in that elephants themselves are composed of elephants and mice at a lower granularity level. We verify the stability of the ranking of streams a few ways: by examing the time-of-day behavior, by calculating the density on the change of rank process over multiple time intervals, and by examining the top elephants and their ranking throughout the day. Our results indicate there exist natural and simple aggregates, based on $\mathrm{p} 8$ and $\mathrm{p} 16$ aggregates, that constitute a large fraction of traffic and that remain stable throughout the day. Many of today's routers provide the capability to do per-prefix load balancing over equal/unequal cost links (e.g., Cisco's Express Forwarding). Load balancing at the traffic granularity that we identify here can be implemented by extending this capability.

\section{RELATED WORK}

Starting with pioneering work by Paxson ([13, 14]), network measurement and monitoring has received widespread attention from Internet researchers and practitioners $([15,3,16,4])$. However, much of this work relies on data collected at public peering points, edge routers and from academic networks. Our work is unique in that the analysis is based on traces and routing tables collected from a operational IP backbone. In this respect, our paper comes closest to the work in $([2,3])$. In [2] Grossglauser et. al. propose a method for following the trajectories of a subset of the packets flowing through a backbone network. The method is based on using a single hash function, based on packet content, at multiple nodes in the network. It has direct applicability to the problem of determining traffic demands on different paths through a network.

Internet measurement data can be broadly divided into routing data and traffic data. The former consists of data about routing protocols and their behaviour. Enormous amounts of such data has been collected and analysed to understand the behaviour of large-scale routing in the Internet $([10,15,13,16])$. Traffic data, consisting of packet traces, is not as widely available, especially from operational backbones. However, both traffic and routing data are need to construct traffic matrices. The use of traffic matrices as a systematic way of representing and analyzing Internet traffic has been gaining attention recently $([3,17,7])$, and [3] is an important recent work in this area. There are strong similarities in the overall goal of the work in [3] and our work - collecting and processing data from an operational backbone in order to understand traffic demands and improve traffic engineering. However our work differs from [3] in a number of ways. First, [3] uses data from peering links at different points in the backbone to construct point-to-multipoint traffic demands across these peering links. These traffic demands comprise only of the transit traffic through their backbone. On the other hand, we collect data from a diverse set of access links (peering, web-hosting, ISPs, etc.) in our backbone, and study the geographic spread of this traffic over the entire backbone. As we showed in this paper, the spatial and temporal characteristics of traffic depends on the type of originating access link; this makes it important to study traffic from different types of access links. Secondly, [3] seeks to build a traffic matrix representing multipoint demands from one router to a set of egress router nodes; this captures all the alternate egress points to a destination network beyond the backbone. In our backbone, I-BGP policies are used to pick one of many egress points to a destination network at any given time. We are interested in studying internal routing and traffic behaviour, given that this egress point has already been determined by I-BGP. Hence we focus only on point-to-point POP-level flows. These differences notwithstanding, both our work and [3] represent important first efforts in understanding backbone traffic demands.

\section{Conclusions}

In this paper, we used packet-level traces collected at a large POP of a tier-1 IP backbone to understand the dynamics of ingress traffic at a backbone POP. In order to study geographical and temporal properties of POP-to-POP traffic, we introduced a methodology for combining our data with BGP information to classify the incoming traffic according to egress POPs. We found that there is a wide disparity in the volume of traffic headed towards different egress POPs. We analyzed the traffic at three granularity levels, the POP level, the access link level, and the destination prefix level. A contribution was to demonstrate different types of temporal stability for each of these on long time scales.

We also examined our network to see how the traffic demands are routed through the backbone. We found that the POP topology and IS-IS link weights are carefully chosen to constrain traffic between most ingress-egress POP pairs to a few paths across the backbone. The combined effect of such routing practices and overprovisioning means that there is a lot of excess capacity in the core that results in (i) underutilized links, (ii) a wide range of link levels within the underutilized range, and (iii) some links being consistenly underutilized. Our findings on the disparate geographic spread of traffic demands combined with current routing practices indicate that there is a lot of room for improvement in load balancing in the backbone. Current routing practices today do not take into consideration the 
traffic demand matrix because such matrices are typically not available. We believe this is one of the key reasons why we see large variation in link load levels.

Our main findings can be summarized as follows.

- The geographic spread of traffic from one ingress POP across all egress POPs is far from uniform. A few egress POPs sink large amounts of traffic, while the majority sink either small or medium amounts of traffic. Our initial assessment of POPs indicates that a simple categorization, in which each category draws about twice the volume of traffic as the one below it (i.e. large/medium and medium/small ratios are approximately two), is possible. Further work needs to be done to model POPs in finer detail. This data is important in that it confirms empirically our intuition (based on internet practices) about how traffic is distributed across backbones. However, it also contradicts the widely used simulation model that assumes uniform distribution of traffic among destination nodes.

- Access links do not distribute traffic similarly across egress POPs; some access links are more likely to send to one set of POPs, while others are more likely to send to a different set of POPs. This differentiation occurs mostly in medium sized egress POPs, and not in large or small POPs.

- We found that the large egress POPs can exhibit a large variability during the day, whereas the medium and small POPs exhibit little variability over a full day. More importantly, we found that when POPs are ranked according to volume, then they maintain their rank throughout the day. With respect to their rank, POPs appear quite stable.

- The elephants and mice phenomenon that we found among streams aggregated on destination prefixes is a natural basis for splitting traffic over multiple paths in the backbone, using routing policies. Identifying reroutable flows at this level of traffic granularity is attractive because such flows exhibit stable behavior throughout the day. Load balancing this way would require early identification of the elephants in the access links of the ingress POPs.

The value of our methodology, observations and analysis extends beyond load balancing to other aspects of backbone engineering. For example, we found a close connection between traffic patterns amongst POPs and the architecture of the POPs themselves. This can help in architecting POPs, or in adding new customers and provisioning backbone capacity when the backbone is upgraded. Also, our analysis of POP behavior, its spatial and temporal characteristics, and its underlying dependence upon access links can be incorporated into capacity planning models.

\section{REFERENCES}

[1] C. Fraleigh, C. Diot, B. Lyles, S. Moon, P. Owezarski, D. Papagianaki, and F. Tobagi, "Design and Deployment of a Passive Monitoring Infrastructure," Passive and Active Measurement Workshop, Amsterdam, Netherlands, April 2001.

[2] N. Duffield and M. Grossglauser, "Trajectory Sampling for Direct Traffic Observation," ACM SIGCOMM, 2000.

[3] A. Feldmann, A. Greenberg, C. Lund, N. Reingold, J. Rexford, and F. True, "Deriving Traffic Demands for Operational IP Networks: Methodology and Experience," ACM SIGCOMM, August 2000.

[4] W. Fang and L. Peterson, "Inter-AS Traffic Patterns and Their Implications," Proceedings of Global Internet, December 1999.

[5] V. Paxson and S. Floyd, "Why We Don't Know How to Simulate the Internet," Proceedings of the 1997 Winter Simulation Conference, December 1997.

[6] D. O. Awduche, A. Chiu, A. Elwalid, I. Widjaja, and X. Xiao, “A Framework for Internet Traffic Engineering," Internet Draft draft-ietf-tewg-framework-02.txt, May 2000.

[7] R. Sabatino, "Traffic Accounting using Netflow and Cflowd," Fourth International Symposium on Interworking, Ottawa, Canada, July 1998.

[8] L. Kleinrock and W. Naylor, "On Measured Behavior of the Arpa Network," AFIPS Conference Proceedings, National Computer Conference, vol. 43, December 1999.

[9] S. Bhattacharyya, C. Diot, J. Jetcheva, and N. Taft, "POPlevel and Access-Link-Level Traffic Dynamics in a Tier-1 POP," Proceedings of ACM Sigcomm Internet Measurement Workshop, November 2001.

[10] G. Huston, “Tracking the Internet's BGP Table," ISMA Winter Workshop, San Diego, USA, December 2000.

[11] S. Nilsson and G. Karlsson, "IP-address lookup using LCtries," IEEE Journal on Selected Areas in Communication, 17(6):1083-1092, June 1999.

[12] B. Fortz and M. Thorup, "Internet Traffic Engineering by Optimizing OSPF Weights,” IEEE Infocom, March 2000.

[13] V. Paxson, "End-to-End Routing Behavior in the Internet," IEEE/ACM Transactions on Networking, vol. 5, pp. 601615, October 1997. 
[14] V. Paxson, "End-to-End Internet Packet Dynamics," ACM SIGCOMM, Cannes, France, September 1997.

[15] K. Claffy, "Internet Measurement and Data Analysis : Topology, Workload, Performance and Routing Statistics," NAE Workshop, 1999.

[16] G. R. Malan C. Labovitz and F. Jahanian, "Internet Routing Instability," ACM SIGCOMM, Canne, France, September 1997.

[17] A. Feldmann, A. Greenberg, C. Lund, N. Reingold, and J. Rexford, "NetScope: Traffic Engineering for IP Networks," IEEE Network Magazine, March 2000.

[18] O. Goldschmidt, "ISP Backbone Traffic Inference Methods to Support Traffic Engineering," 2nd ISMA Winter Workshop, San Diego, CA., December 2000.
[19] S. V. Wiel, J. Cao, D. Davis, and B. Yu, “ Time-varying Network Tomography: Router Link Data," Symposium on the Interface: Computing Science and Statistics, June 1999.

[20] B. Chinoy, "Dynamics of Internet Routing Information," ACM SIGCOMM, 1993.

[21] A. Shaikh, J. Rexford, and K. Shin, "Load-Sensitive Routing of Long-Lived IP Flows," ACM SIGCOMM, pp. 215226, September 1999.

[22] A. Sridharan, S. Bhattacharyya, C. Diot, R. Guerin, J. Jetcheva, and N. Taft, "On the Impact of Aggregation on the Performance of Traffic Aware Routing"," Proceedings of the 17th International Teletraffic Congress (ITC), Salvador do Bahia, Brazil, September 2001. 